\title{
Vagal modulation of 1-month-old infants to auditory stimuli is associated with self-regulatory behavior
}

\author{
Sara Cruz ${ }^{1}$ (1) | Fernando Ferreira-Santos ${ }^{2}$ (1) | \\ Patrícia Oliveira-Silva1,3 | Eugénia Ribeiro ${ }^{1}$ | Óscar Gonçalves ${ }^{1,4,5}$ \\ Adriana Sampaio ${ }^{1}$
}

\footnotetext{
${ }^{1}$ University of Minho

${ }^{2}$ University of Porto

${ }^{3}$ Universidade Católica Portuguesa

${ }^{4}$ Northeastern University

${ }^{5}$ Harvard Medical School

\section{Correspondence}

Sara Cruz, Neuropsychophysiology Laboratory, School of Psychology, University of Minho, Campus de Gualtar, Braga, Portugal.

Email: saracruz@psi.uminho.pt

Funding information

Fundação Bial, Grant/Award Number: 42/ 08; Fundação para a Ciência e Tecnologia (FCT), Grant/Award Number: SFRH/BD/ $68263 / 2010$

\begin{abstract}
During infancy, cardiac vagal modulation has been associated with attentional and social engagement behaviors. While studies have shown that infants display a behavioral repertoire that enables them to interact with others by being able to regulate themselves in order to attend to and to discriminate emotional and social cues, vagal modulation to sensory stimuli and its association with behavioral outcomes at early ages remains to be addressed. In this study, we analyzed the cardiac vagal response of 1-month-old infants to two auditory stimuli intensities and whether vagal response was associated with social interactive and self-regulatory abilities. Therefore, we recorded cardiac and respiratory physiological responses in 28 infants using a Biopac System. Neurobehavioral assessment was performed using the Neonatal Behavioral Assessment Scale. We observed increased respiratory sinus arrhythmia (RSA) amplitude to both auditory stimuli intensities when compared to baseline. No intensity effect was found for the RSA response. Additionally, we observed that higher RSA amplitude to both auditory stimuli was positively correlated with adjusted self-regulatory behaviors, suggesting a convergence between multiple measures assessing infants' state regulation. Results are discussed in light of 1-month-old infants' auditory stimuli processing and its implications for regulatory behaviors and the emergent social-like behaviors.
\end{abstract}

\section{KEYWORDS}

auditory processing, infants, neurodevelopment, orienting behavior, self-regulatory behavior, vagal modulation 


\section{1 | INTRODUCTION}

Since birth, infants are able to interact with others through a behavioral repertoire that depends on adequate physiological regulation and adjustment, as young infants need to be capable of successfully adapting and regulating themselves in order to behaviorally attend and respond rapidly to the environment (Brazelton \& Nugent, 1995; Porges, 2011).

This successful adjustment has also been associated with other adequate behavioral interactive routines and attentional responses (e.g., interested behavior, gazing, lower motor activity, or engagement with people and objects) that the newborn displays to the caregiver and the environment (Als, 1986; Als, Butler, Kosta, \& McAnulty, 2005; Bazhenova, Plonskaia, \& Porges, 2001; Figueras et al., 2011; Lundqvist \& Sabel, 2000; Perez-Pereira et al., 2013). These regulatory and adjustment strategies for social engagement, which can be understood as infants' ability to modulate their behavior in order to engage and respond to environmental interactions, depend on a visceral state that depends on the nervous system (Porges, 1992, 1995, 2001, 2003).

The Polyvagal Theory (Porges, 1995, 2001) suggests that two pathways of the vagus nerve mediate infants' reactivity to the social environment, by either (a) reducing cardiac output and thereby promoting calm states and social involvement (vagal brake), or (b) increasing cardiac output and producing mobilization behaviors, thereby avoiding social involvement (withdrawal of vagal brake). These pathways are components of the Social Engagement System (Porges, 2003) and together are considered a putative biological basis underlying the emergence of social-oriented behaviors in infants. Furthermore, these pathways have an impact on later social behavioral outcomes (Porges \& Furman, 2011). In fact, a decrease in respiratory sinus arrhythmia (RSA) amplitude, which reflects cardiac activity and is a measure of the dynamic regulation of the myelinated vagus, has been associated with mobilization behaviors (defensive reflex), whereas an increase in RSA amplitude, which is though to reflect the parasympathetic influence in the heart, has been associated with social engagement behaviors (orienting reflex) (Porges, 2001).

Indeed, there is evidence that distinctive cardiac activity patterns may underlie newborns' attentional or avoidance behaviors to different sensorial stimuli (Clarkson \& Berg, 1983; Davis, Crowell, \& Chun, 1965; Posner, 1995; Richards, 2001; Vranekovic, Hock, Isaac, \& Cordero, 1974). In particular, attentional competences, which are behavioral manifestations of social engagement, have been highly associated with self-regulatory and emotional reactivity control accompanied by enhanced inhibitory control over motor behavior (Calkins, 1997; Rueda, Posner, \& Rothbart, 2004). This behavioral pattern emerges in infancy and is under the control of the parasympathetic system (Porges, 2003).

Specifically, young infants' behavioral and physiological functioning with regard to environmental interactions are reflected in their threshold to attend to visual and auditory stimuli of distinct intensities (Calkins, Fox, \& Marshall, 1996). Of particular interest for social development is auditory processing, that is, the infant's ability to attend to sounds and voices.

Newborns seem to respond with increased heart rate acceleration to auditory stimuli at onset followed by decreased cardiac output after repetition (Field, Dempsey, Hatch, Ting, \& Clifton, 1979). Porges, Arnold, and Forbes (1973) presented a moderately intense auditory stimulus for 30s to two groups of newborns; one group displaying high heart rate variability, and the other low heart rate variability. The authors found the high variability group to present cardiac acceleration to stimuli onset, deceleration to offset, and decreased variability during stimuli presentation, while the low variability group presented an attenuated acceleration to stimuli onset. This suggests that both groups were able to habituate to the auditory stimulus and consequently attend to it. In this sense, the authors showed that cardiac response is a reliable measure of indexing attentional responsivity in newborns. More recently, the cardiac activity response was found to be associated with perception of acoustic stimuli related to the human voice and emotion, showing that young infants display differential psychophysiological responses when attending to particular voice cues (e.g., infant direct [ID] speech) (Grossmann, Striano, \& Friederici, 2005; Lewis, Stanfill, Zageris, Davila, \& Porges, 2011) and to the affective emotional state of the ID speech (Santesso, Schmidt, \& Trainor, 2007).

Physiological regulation to auditory stimuli may convey important information regarding the necessary stimuli intensity to produce positive social engagement behaviors. In accordance, Lundqvist and Sabel (2000), verified that 
healthy infants who had difficulties in self-regulation presented difficulties in social interaction with the examiner, which compromised their ability to learn from the environment and, consequently, the ability to acquire distinct social and developmental competences. Further support to this hypothesis is presented by Perez-Pereira et al. (2013) who evidenced that environmental factors were closely associated with orientating and self-regulatory abilities in full-term, healthy infants. Among these, social factors were more predictive of behavioral outcomes, such as attention abilities.

Overall, studies emphasize the role of physiological mechanisms involved in the perception of auditory stimuli during infancy. It is possible that these mechanisms are translated as listening skills as they improve in parallel with adjusted state regulation and are implicated in the emergence of early social-oriented behaviors (Porges \& Lewis, 2010; Porges et al., 2013). In accordance, basal RSA was found to be associated with individual differences in arousal (Beauchaine, 2001; Hastings et al., 2008). Indeed, typically developing children presented higher basal RSA amplitude that was further related with emotional reactivity. This physiological pattern was different from those children presenting emotional regulation difficulties (Beauchaine, 2001; Hastings et al., 2008). In addition, the authors suggested that dynamic changes in vagal modulation mirror an active regulation of arousal in order to support effective coping strategies regarding environmental and social challenges (Beauchaine, 2001; Hastings et al., 2008). That is, the cardiac regulation by the vagus could enhance and promote calm and positive social engagement (Porges, 1995).

Taken together, in this study we investigated baseline and vagal modulation of typical 1-month-old infants to two auditory stimuli intensities (lower and higher) and its relation with orienting and self-regulatory abilities. Building on the evidence provided by the Polyvagal Theory (Porges, 1992), in this study we assume that vagal modulation is related to attention to auditory stimuli presented in two different auditory intensities. In addition, we hypothesize that vagal modulation to these stimuli intensities is differentially related to social-like behaviors in young infants.

Previous evidence suggests that differential neurophysiological responses seem to be associated with the processing of distinct auditory stimulus, which is translated into different behavioral characteristics (Kushnerenko et al., 2013; Porges et al., 2013). Newborns and young infants are reported to show an increased sensitivity to low intensity auditory stimulus, displaying consequently more attentional behaviors (Kushnerenko et al., 2013). In accordance, electrophysiological evidence proposes distinct neural responses for processing distinct acoustic stimuli in typical development, which is particularly relevant for learning and language development (Kushnerenko et al., 2013). Building on this evidence and on Porges et al. (2013) proposal suggesting that state regulation and auditory processing are dependent on the same physiological system, we aimed to investigate if distinct auditory stimuli (auditory intensities) would reflect better physiological regulation and, consequently, adjusted social-like behavioral outcomes.

Since neither auditory intensities constitute aversive stimuli, we expected (a) less vagal withdrawal (i.e., vagal augmentation translated as higher RSA amplitude) to both low and high auditory intensities compared to baseline RSA, representing better coping strategies in order to respond adequately to different social contexts (Porges, 2003; Hastings et al., 2008; Schmidt \& Segalowitz, 2008). Nevertheless, less vagal withdrawal (higher RSA amplitude) to lower stimulus intensity-compared to higher stimulus intensity-is expected, since infants seem to present increased sensitivity to sounds with low intensity (Kushnerenko et al., 2013). In addition, we expect that (b) less vagal withdrawal will be associated with better self-regulatory abilities, which are translated behaviorally into attentive states.

\section{METHODS}

\subsection{Participants}

This study was reviewed and approved by the ethical committee of the hospital where the study was conducted. We recruited mother/infant dyads when the infants were born. All infants were Caucasian, born full-term, and healthy at the moment of data collection. Psychophysiological measures were recorded for 28 one-month-old infants. Three infants did not complete the neurobehavioral assessment as its application was discontinued because of the infants' distress. Therefore, the total number of participants that received physiological assessment was 28 infants (12 female), and neurobehavioral assessment was 25 (12 female) (Table 1). All participating families gave their written informed consent in accordance with the Declaration of Helsinki. 
TABLE 1 Infant's health information at the time of recruitment and collection

$\begin{array}{llllc} & \text { Infants characteristics } & \text { Mean } & \text { SD } & \text { Min-max } \\ \text { At recruitment time } & \text { Gestational Age (weeks) } & 39 & 1.3 & 37-41 \\ & \text { Weight (grams) } & 3210 & 360 & 2,590-3,900 \\ & \text { Height (centimeters) } & 48.8 & 1.7 & 46-52 \\ & \text { Apgar Score (10th min.) } & 10 & 0 & 10-10 \\ \text { At collection time } & \text { Age (days) } & 34 & 7.3 & 18-45 \\ & \text { Total Infants with physiological assessment } & 28 & 25 & \\ & \text { Total Infants with physiological and NBAS assessments } & 25 & \end{array}$

\section{2 | Neurobehavioral assessment}

We used the neonatal behavioral assessment scale (NBAS) for the neurobehavioral assessment of the infants (Brazelton \& Nugent, 1995). A trained and reliable examiner performed the infants' assessment and coding process. NBAS is used to characterize behavioral profiles of infants. It assesses the infant's behavioral repertoire through 28 behavioral items coded on a 9-point scale and the neurological state through 18 reflex items coded on a 4-point scale. It is organized around seven domains (Lester, Als, \& Brazelton, 1982): Habituation, Orientation, Motor System, Range of State, Regulation of State, Autonomic Stability, and Reflexes. Two of these domains are considered to reflect infants' social behavior (Brazelton \& Nugent, 1995): Orientation and Regulation of State. Orientation assesses the infant's ability to visually follow an object (inanimate) and the examiner's face (animate) and to auditory follow an object sound (inanimate) and the examiner's voice (animate). It also assesses the quality of alertness, as the infant needs to be in a quiet, alert state in order to successfully interact with the environment. Orientation allows the classification of infants' orienting behavior and interactive abilities concerning their response to external stimuli. Regulation of State assesses the infant's ability to calm down and maintain their attention to stimuli and, therefore, reflects his/her behavioral selfregulatory abilities (Als, Tronick, Lester, \& Brazelton, 1977; Sprangler, Fremmer-Bombik, \& Grossmann, 1996).

We considered all NBAS items in order to characterize each infant's behavioral profile. However, for the purposes of this study, we selected Orientation and Regulation of State domains. Both domains are assumed to reflect adjusted social-like characteristics in young infants since orienting (behavioral interactive characteristics) and regulatory abilities are suggested to be basic competences associated with the infant's ability to attend to and modulate their behavior in order to respond adequately to the surrounding environmental interactions (Brazelton \& Nugent, 1995; Calkins, 1997; Cruz, Crego, Ribeiro, Goncalves, \& Sampaio, 2015; Sprangler et al., 1996). We used the vagal response as a physiological marker correlated with adjusted social behavior in young infants (higher scores in these NBAS domains) since these two domains are associated with social features displayed by very young infants (Brazelton \& Nugent, 1995; Lester et al., 1982) and the RSA measure is frequently used as an indicator of social engagement (Porges, 2003).

Specifically, we computed the mean Orientation score considering the items inanimate visual, inanimate auditory, inanimate visual-auditory, animate visual, animate auditory, animate visual-auditory, and alertness. Similarly, we computed the values obtained in the items cuddliness, consolability, self-quieting, and hand-to-mouth as the mean score of Regulation of State. Higher punctuations reflected typical behavioral performance in both domains. All infants were in a quiet alert state at the time of assessment and only their best performance was considered for the coding process.

\section{3 | Auditory stimuli}

Two simple tone auditory stimulus intensities were created in Audacity software (version 1.3.6 for OS X, www.audacityteam.org) at $900 \mathrm{~Hz}$ frequency: a low intensity $(50 \mathrm{~dB})$ and a high intensity $(70 \mathrm{~dB})$. The high intensity was selected first, as it is often used in developmental studies, and the lower intensity was generated by reducing the sound pressure level by $20 \mathrm{~dB}$ (Schmidt \& Segalowitz, 2008). Each stimulus was a click-like sound with a duration of $100 \mathrm{~ms}$ and a 900 ms interstimulus interval, corresponding to epochs lasting 1 s. Presentation ${ }^{\circledR}$ software (Version 0.61.3, www. 
neurobs.com) delivered the auditory stimuli. Stimulus delivery was organized in a block paradigm according to the auditory stimulus intensity: one block consisted of sixty $50 \mathrm{~dB}$ auditory stimuli and the other block of sixty $70 \mathrm{~dB}$ stimuli. Each block was presented once during 1 min, 1 stimulus per second, with a 20-s interval between blocks. The order of block presentation was counterbalanced across participants.

\subsection{Physiological assessment and signal processing}

Before recording cardiac activity, each infant's chest was cleaned with distilled water and, then, three pre-gelled hypoallergenic $\mathrm{Ag} / \mathrm{AgCl}$ electrodes were positioned at the right, left, and medial zones of the chest, in a modified Lead II electrode configuration (Schmidt \& Segalowitz, 2008). For respiration recording, a respiratory band was placed around the infants' stomach as infants' respiration occurs mainly at the abdominal level (Schmidt \& Segalowitz, 2008). To ensure the quality of the signal, the online trace was visually examined before starting the recording. A 5-min baseline was recorded and, afterwards, the auditory stimuli were delivered. Recordings were conducted with Biopac MP-150 equipment (Biopac System, Santa Barbara, CA, USA), coupled with ECG100C and DA100C modules for registration of cardiac and respiratory activities, respectively. The Biopac amplifier was connected to a computer equipped with Acknowledge software (Biopac Systems) and was used to define the acquisition parameters, and store and preprocess the physiological data. Physiological signals were amplified 1,000 times with a bandpass filter of 0.05-100 Hz, then digitized at $500 \mathrm{~Hz}$.

We used the QRSTool/CMetX software (Allen, Chambers, \& Towers, 2007) (version 1.2.2.) for physiological data analysis. Electrocardiogram (ECG) recordings were exported from Acknowledge into QRSTool for automatic R peak detection and calculation of the inter-beat interval (IBI) series. The results of the automatic scoring procedure were visually inspected to screen for the misidentification of R peaks and errors were corrected by manually scoring the actual R peak. Finally, the IBI series were exported to CMetX where several metrics of cardiac activity were computed. In the present study, we focused on RSA (Allen et al., 2007). RSA was calculated as the log variance of the IBI series after bandpass filtering in the canonical infant respiratory frequency band of $0.24-1.04 \mathrm{~Hz}$ (Allen et al., 2007). Visual inspection of the respiratory power spectra (obtained using in-house scripts in MATLAB-The MathWorks, Natick, MA, USA) ensured that the peak respiratory frequency of all infants was included in the interval used for RSA filtering (Berntson et al., 1997).

We extracted the first good 1-minute of data from the 5-min baseline collected for the statistical analysis so that its duration would be equivalent to the duration of each auditory stimulus block. Afterwards, we adjusted the physiological values in each condition (lower and higher intensity) to baseline by subtracting the auditory stimuli raw score from the baseline score [ASx (where ' $x$ ' stands for auditory stimuli intensity 1 or 2)-BA (1-min baseline)]. We did so because previous literature has demonstrated that dynamic changes in vagal modulation, contrary to basal RSA, better reflects infants' active regulation of arousal to support effective coping and attentional control over social situations (Calkins, Graziano, \& Keane, 2007; Hastings et al., 2008).

\section{$2.5 \mid$ Procedure}

We performed data collection in a room with temperature around $20-25^{\circ} \mathrm{C}$, with controlled luminosity and sound. As the family arrived, we explained the study objectives and procedures and obtained the informed consent. Infant data collection either started with neurobehavioral assessment or psychophysiological recordings, depending on the infant's state, as the NBAS Habituation domain can only be administered if the infant is sleeping. The Habituation domain assesses infants' ability to shut out visual, auditory, and tactile stimuli and keep themselves in a sleeping state. Therefore, if the infant arrived asleep we would start with the administration of the NBAS, and on the contrary, if the infant was awake we would start with the physiological assessment, allowing the infant to fall asleep thereafter. About 18 of the 28 infants were asleep and 10 were awake during physiological assessment (in both baseline and auditory stimuli recording). We considered those infants who had closed eyes, regular respiration, and no or little spontaneous body movements (either in deep or active sleep) as being in a sleeping state; awake infants were characterized as having a bright look directed to the stimuli, minimal motor activity, and reactive to the stimuli (in this category, we also included infants that were irritable during the stimuli presentation) (Brazelton \& Nugent, 1995). 
TABLE 2 RSA mean amplitude and standard deviation values of baseline and auditory intensity conditions

\begin{tabular}{lll} 
& RSA & Standard deviation \\
\cline { 2 - 3 } & Mean & 1.3 \\
Baseline & 3.1 & 1 \\
\hline Lower intensity & 3.5 & 1 \\
\hline Higher intensity & 3.2 & 1 \\
\hline
\end{tabular}

An ECG and respiratory rates were recorded for the physiological assessment. Mothers sat in a comfortable chair with the infant placed on her lap. Mothers were asked to remain quiet and to not move themselves or the infant during the session. Two speakers held by a researcher at a distance of approximately $20 \mathrm{~cm}$ from each of the infant's ears delivered the auditory stimuli. Using the pacifier was the maneuver recommended for calming the infant down, and when this was necessary the session was paused. The overall duration of the data collection (physiological and neurobehavioral assessments) was approximately $30 \mathrm{~min}$.

\subsection{Statistical data analysis}

Statistical analysis was performed using IBM SPSS Statistics 22. Repeated measures of Covariance (ANCOVAs) were conducted with measurements of RSA amplitude (adjusted to baseline) with intensity stimulus (low and high) as the withinsubject factor to verify the effect of auditory stimuli intensity on RSA response. Infants' gestational age, sex, weight, height, state and age at data collection were inserted as covariates in order to test their effect on the physiological response. An alpha level of 0.05 was used and, whenever appropriate, degrees of freedom were corrected by the conservative GreenhouseGeisser estimate. All post hoc paired comparisons were performed with the Bonferroni adjustment for multiple comparisons.

NBAS items' mean and standard deviation were computed in order to behavioral characterize our sample.

Further analyses were performed to examine the correlation between physiological response (baseline RSA and low-intensity and high-intensity RSA) and neurobehavioral Orientation and Regulation of State domains. Linear regression analyses were conducted, as we defined RSA metric as our Independent factor. Gestational age, sex, weight, height, infants' state and age at data collection variables were also inserted to verify their effect in our regression model. We defined Orientation and Regulation of State scores as our dependent factor.

\section{3 | RESULTS}

Infant's baseline RSA, low-intensity RSA and high-intensity RSA amplitude values are presented in Table 2. NBAS analysis revealed that all infants displayed organized orienting, regulatory and motor controlled behavior (Table 3). Correlations between demographic, physiological measures and NBAS data are reported in Table 4.

TABLE 3 Mean and standard deviation values of NBAS scale

\begin{tabular}{lll}
\hline NBAS & Mean & Standard deviation \\
\hline Habituation & 7 & 2.5 \\
\hline Orientation & 7.6 & 0.9 \\
\hline Motor system & 5.9 & 1.5 \\
\hline Range of states & 3.8 & 1.5 \\
\hline Regulation of states & 5.7 & 2.7 \\
\hline Autonomic system & 4.3 & 1.6 \\
\hline Reflexes & 1.9 & 0.3 \\
\hline
\end{tabular}




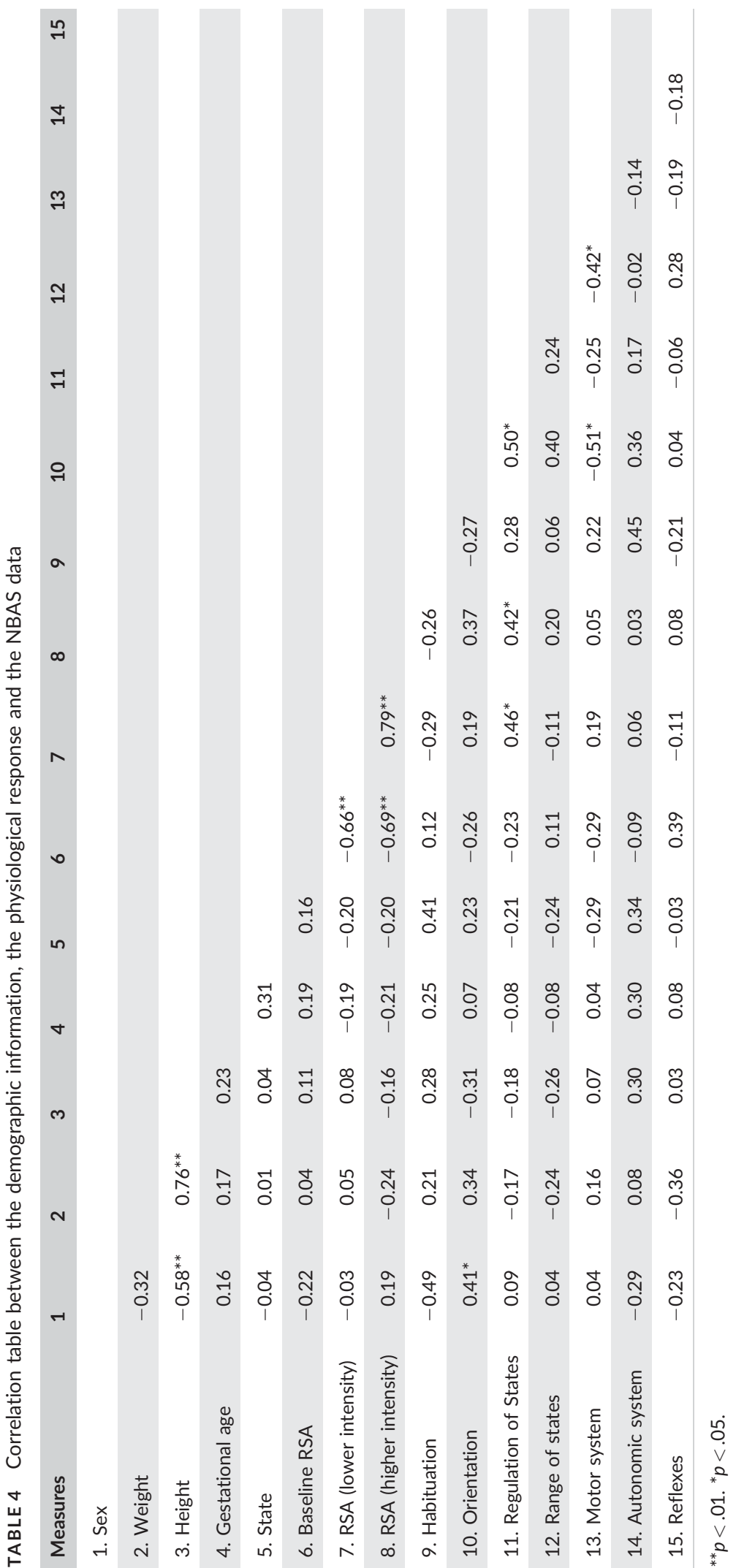


TABLE 5 Linear regression model: predictors of Orienting and Regulation of State behaviors

\begin{tabular}{|c|c|c|c|c|c|c|}
\hline & \multicolumn{3}{|c|}{ Orientation } & \multicolumn{3}{|c|}{ Regulation of sate } \\
\hline & $\boldsymbol{\beta}$ & $t$ & $p$ & $\boldsymbol{\beta}$ & $t$ & $p$ \\
\hline \multicolumn{7}{|l|}{ Lower intensity } \\
\hline RSA & 1.93 & 0.95 & 0.35 & 0.46 & 2.5 & $0.02^{*}$ \\
\hline Gestational age & -0.02 & 0.08 & 0.94 & 0.07 & 0.25 & 0.81 \\
\hline Sex & 0.42 & 1.46 & 0.16 & -0.2 & -0.66 & 0.52 \\
\hline Weight & 0.34 & 1.19 & 0.25 & 0.006 & 0.02 & 0.99 \\
\hline Height & 0.14 & 0.35 & 0.73 & -0.41 & -1.005 & 0.33 \\
\hline State & 0.28 & 1.21 & 0.24 & -0.03 & -0.11 & 0.92 \\
\hline Age at data collection & -0.12 & 0.47 & 0.65 & 0.2 & 0.74 & 0.47 \\
\hline \multicolumn{7}{|l|}{ Higher intensity } \\
\hline RSA & 0.37 & 1.88 & 0.07 & 0.42 & 2.23 & $0.04^{*}$ \\
\hline Gestational age & -0.04 & -0.17 & 0.87 & 0.03 & 0.12 & 0.9 \\
\hline Sex & 0.37 & 1.19 & -0.24 & -0.28 & -0.92 & 0.37 \\
\hline Weight & -0.24 & -0.81 & 0.43 & 0.13 & 0.42 & 0.68 \\
\hline Height & 0.1 & 0.25 & 0.81 & -0.45 & -1.09 & 0.29 \\
\hline State & 0.3 & 1.3 & 0.21 & -0.002 & -0.007 & 0.99 \\
\hline Age at data collection & -0.016 & -0.07 & 0.95 & 0.34 & 1.36 & 0.19 \\
\hline
\end{tabular}

${ }^{*} p<.05$.

\subsection{RSA response to auditory stimuli}

The ANCOVA analysis indicated that auditory stimuli intensities did not have an effect on RSA response, $F(1,27)=2.8$, $p=.10, \eta_{\mathrm{p}}{ }^{2}=0.094$ (low-intensity RSA response: $M=0.35, S D=1.3$; high-intensity RSA response: $M=0.07, S D=1.3$ ). Similarly, infant's gestational age, $F(1,21)=.24, p=.63, \eta_{p}{ }^{2}=0.011$, sex, $F(1,21)=2.39, p=.14, \eta_{p}{ }^{2}=.102$, weight, $F(1$, $21)=3.87, p=.06, \eta_{p}{ }^{2}=0.155$, height, $F(1,21)=0.32, p=.58, \eta_{p}{ }^{2}=0.015$, and state, $F(1,21)=0.4, p=.54, \eta_{p}{ }^{2}=0.018$, had no effect on RSA response. An effect of infants' age at the moment of data collection was found on RSA response, $F(1$, $17)=6.21, p=.02, \eta_{\mathrm{p}}{ }^{2}=0.228$. No significant differences were observed between the auditory intensities $(p=.07)$.

\subsection{Association between physiological and neurobehavioral assessments}

Regression analysis showed that low-intensity and high-intensity RSA response did not predict orienting behavior of 1month-old infants (Table 5). RSA amplitude did not explain a significant amount of variance in the Orientation domain score in both low-intensity RSA response, $R^{2}=.04, F(1,24)=.89, p=.35$, and high-intensity $\mathrm{RSA}$ response, $R^{2}=.13, F$ $(1,24)=3.55, p=.07$. However, RSA response significantly predicted self-regulatory abilities in 1-month-old infants regarding low-intensity and high-intensity auditory stimuli, as illustrated in Figure 1 (see also Table 5). RSA response explained a significant amount of variance in the State Regulation domain, again regarding both low-intensity RSA response, $R^{2}=.21, F(1,24)=6.23, p=.02$, and high-intensity $R S A$ response, $R^{2}=.18, F(1,24)=4.95, p=.04 .^{1}$

Infants' gestational age, sex, weight, height, state, and age at the moment of data collection did not predict orienting behaviors in either low-intensity or high-intensity RSA response (Table 5) and did not explain the amount of variance in the Orientation domain score, neither in the low-intensity RSA response, $R^{2}=.37, F(7,24)=1.47, p=.25$, nor in the high-intensity RSA response, $R^{2}=.3, F(7,24)=.48, p=.82$. Similarly, the same variables did not predict selfregulatory behaviors, in the low-intensity or in the high-intensity RSA response (Table 5) and they also did not explain the amount of variance in the Regulation of State domain score in the low-intensity RSA response, $R^{2}=.31, F$ $(7,24)=.86, p=.82$, or in the high-intensity RSA response, $R^{2}=.37, F(7,24)=1.07, p=.41$.

\section{4 | DISCUSSION}

In the present study, we investigated RSA responses of 1-month-old healthy infants to two auditory stimulus intensities. Additionally, we analyzed the correlation between RSA response amplitude, considering both auditory stimulus 

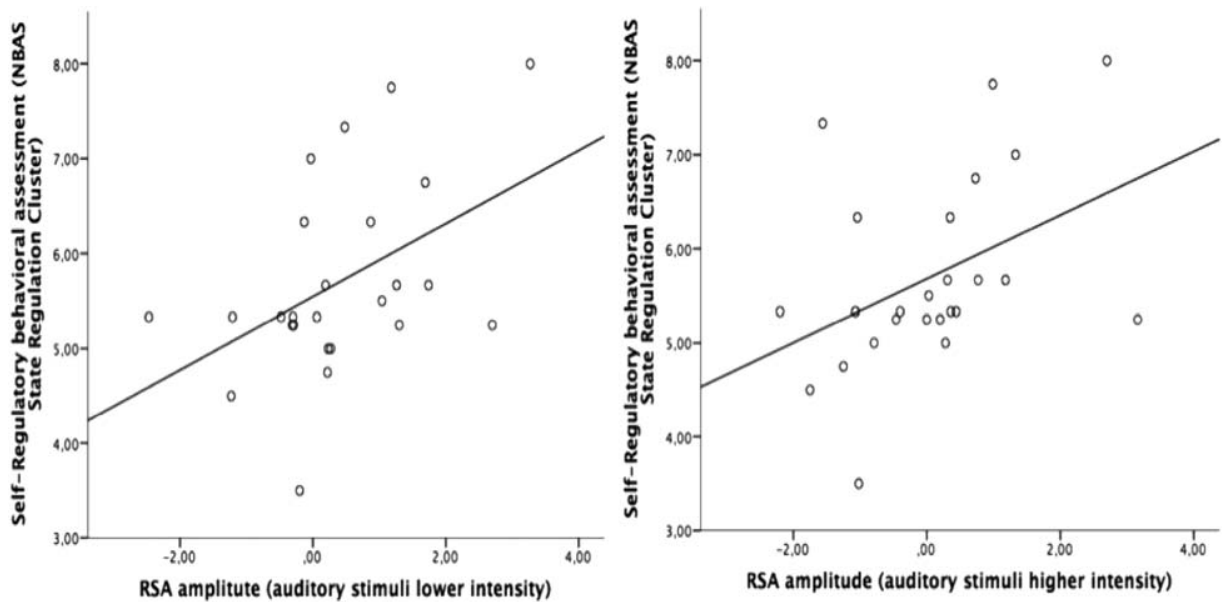

FIGURE 1 Regression analyses scatterplots depicting: (Left) association between RSA amplitude in the lower auditory stimulus intensity and State regulation NBAS cluster; (Right) association between RSA amplitude regarding the higher auditory stimulus intensity and State regulation NBAS cluster

intensities, and neurobehavioral assessment, specifically with orienting and self-regulatory abilities, using the Orientation and Regulation of State NBAS domains. We computed separated models considering both intensities separately because we were interested in verifying which auditory intensity would be associated with better physiological response and with adjusted social/behavioral abilities. Since infants were full-term and healthy, we expected that less vagal withdrawal to both stimuli would reflect better response to social contexts, translated into more adequate selfregulatory abilities, as previously suggested by Hasting et al. (2008).

Our results showed that 1-month-old infants presented increased RSA amplitude to both auditory stimulus intensities when compared to the baseline, suggesting that, at a very early age, infants seem to be able to attend to distinct auditory stimulus intensities and, consequently, display attentional and orienting behaviors to them. This seems to be corroborated by the neurobehavioral assessment (NBAS behavioral profile) considering that increased self-regulatory abilities and decreased motor activity are both correlated with adjusted orienting behaviors, which may be underlying attentive behavior (Table 3) (Calkins, 1997; Rueda et al., 2004). Additionally, we did not find an effect of auditory stimuli intensity on the RSA measure suggesting that infants attend to both intensities equally (shown by the increased RSA amplitude to both lower and higher intensities). This may be due to similar physical proprieties of the auditory stimuli $(\mathrm{dB})$ and, therefore, infants seem to respond similarly to both intensities. In this sense, our results suggest that both stimulus intensities may promote attentional behaviors. In fact, young infants seem to display attention to low auditory intensity sounds, as previously suggested (Kushnerenko et al., 2013). Indeed, this may occur due to the fact that during the first months of life (until circa 3 months of age) infants present an increased sensitivity to sounds with low intensity, which is observed through their behavior (e.g., displaying more looking directed to the stimulus) (Kushnerenko et al., 2013).

Moreover, it has been argued that increased RSA in regards to processing low frequency sounds reflects the middle ear function of absorbing acoustic frequencies that may be necessary to distinguish features of vocalizations and, again, is associated with attentive behavioral states (Lewis et al., 2011). According to the Polyvagal Theory (Porges \& Furman, 2011; Porges et al., 2013), auditory processing may predict state regulation characteristics. The downregulation of neural influences on the heart (via the vagus nerve) and to the middle ear muscles (via the facial and trigeminal cranial nerves) allows orienting and attentional behaviors (Porges, 2003). In this sense, our results seem to be in line with the authors' findings showing that infants' improved physiological self-regulation toward auditory intensity stimuli may underlie attentional and orienting behaviors, independently of the stimuli intensity.

Besides this evidence, it is particularly interesting to highlight that both auditory intensities are quite similar to the speech sound intensity produced in a quiet conversation (when compared to a normal conversation tone) and is usually 
verified in ID speech. ID speech conveys specific characteristics that enhance communication of positive affect, modulates infants' arousal, and elicits and maintains their attention to the environment (Bryant \& Barrett, 2007; Fernald, 1993; Grossmann et al., 2005). In this sense, our results seem to reflect that, very early in development, higher RSA amplitude to auditory stimuli may index physiological regulation abilities. This occurs through vagal modulation, allowing infants to attend to stimuli and, consequently, acts as a platform supporting later social and communication abilities (Porges, 2001, 2003).

In accordance, auditory processing may be assumed as a predictor of regulatory abilities at a very early age as mirrored by vagal regulation toward low and high auditory stimulus intensities (Porges et al., 2013). However, we should draw this conclusion with caution as our results are not significant and this study must be replicated with a higher number of participants.

However, differences in RSA amplitude to distinct auditory intensities are associated with infants' age. Infants' physiological self-regulatory behaviors and, consequently, attentional states, may be associated with maturational improvement, which is highly associated with age (Porges, 2001, 2003). It has been shown that strategies for social adjustment rely on a supportive visceral state regulated by the autonomic nervous system that allows for social interaction (Porges, 1992, 1995). In this sense, self-regulatory behaviors and, thus, the regulation of visceral states, are dependent on the maturational processes of the vagal pathways (Porges, 1992). These pathways affect cardiac output by either promoting calm or mobilization behaviors, which are associated with autonomic functions (i.e., regulation of visceromotor and somotomotor components) to adjust physiological states and support social interactions (Porges, 2001). In this sense, higher RSA amplitude to both auditory stimuli may suggest adaptive physiological regulation abilities, which are in turn related to infants' age. Our findings corroborate the large body of evidence regarding vagal modulation (Porges, 1992, 1995, 2001, 2003) and may be of particular interest for the study of vagal modulation maturation processes and its association with social behaviors at early ages. We, therefore, suggest that further investigation is required.

Additionally, our findings show a positive correlation between low-intensity and high-intensity RSA response and Regulation of State domain, suggesting that 1-month-old infants who physiologically regulate themselves display more adjusted self-regulatory behaviors.

Previous studies have shown that engagement or mobilization behaviors seem to have a physiological basis, since it has been proven that vagal regulation is associated with different behavioral outcomes across infancy (Beauchaine, 2001; Porges \& Furman, 2011; Propper \& Moore, 2006). In this sense, our study accounts for the evidence that healthy infants who physiologically regulated themselves to both low and high auditory stimulus intensities display adjusted social-like behaviors. This is supported by the fact that RSA amplitude in both auditory intensities is associated with behavioral regulatory abilities, suggesting that 1-month-old infants are already able to physiologically regulate themselves in order to attend to auditory information. As referred to previously, improved state regulation is often observed as an increased ability to sustain attention while actively inhibiting responses to distracters (Porges et al., 2013). Our results are consistent with previous findings showing that 1-month-old infants' vagal modulation is underlying their ability to regulate their behavioral state in order to attend to auditory information, since infants' increased RSA to auditory stimuli underlies orienting and attending behaviors (Porges, 2011),

This finding seems particularly interesting when considering the importance that interactive exchanges play in infants' development. At an early postnatal age, infants are able to regulate themselves physiologically and behaviorally, and can therefore attend to auditory information. This ability seems to support adaptive interactive routines and attentional responses to the environment and caregivers through calm and organized behaviors (Figueras et al., 2011; Lundqvist \& Sabel, 2000; Perez-Pereira et al., 2013; Porges, 1992). Various studies have shown that vagal modulation is essential for promoting social interactive behaviors in young infants as it has an important role in attenuating sympathetic system influences at the level of the heart and allowing social communication (Porges \& Furman, 2011).

In particular, our results show that vagal modulation at an early age in development seems associated with neurobehavioral regulatory ability, which in turn may underlie positive social-like interactive exchanges such as orienting and attentional behaviors. As described in Polyvagal theory, the social engagement system is built on self-regulatory 
abilities that allow infants to interact with the environment (Porges, 2003). Accordingly, vagal modulation of 1-monthold infants may reflect a physiological marker of infants' neurobehavioral adjustment. This modulation is particularly associated with self-regulatory abilities, which seems to underlie adjusted social-like behaviors.

The major limitation of this study concerns the small sample size. Thus, this study should be replicated with a larger sample in order to confirm our findings. Likewise, we did not find statistically significant results concerning the baseline RSA, which has been previously demonstrated to be important for understanding individual differences regarding physiological reactivity (Hastings et al., 2008). This lack of differences may also be due to our small sample size and, therefore, future studies with a larger sample should be conducted. Moreover, the lack of racial diversity is another limitation since it has been shown that parasympathetic functioning differs across different racial and ethnic groups (Hill et al., 2015). Future studies should address processing of other sensorial stimulus intensities, such as visual or olfactory, and its association with neurobehavioral outcomes in young infants. Furthermore, the impact of vagal modulation of young infants on future developmental outcomes (cognitive or language abilities) should be investigated.

\section{ACKNOWLEDGMENTS}

Foremost, we thank all the families and infants who participated in this study. We would like to thank Pedro Hispano Hospital, particularly to Dr. J. Lopes dos Santos, for all the kindness throughout the time that we were there for the data collection process. We thank Maria de Góis-Eanes for all the help and knowledge and Santiago Galdo-Álvarez for the contributions to this study. Furthermore, we thank Associação Viver a Ciência for the Simbiontes prize in 2013. This work was supported by Fundação Bial (grant number 42/08) and financed by Fundação para a Ciência e Tecnologia (FCT) under a PhD grand (reference SFRH/BD/68263/2010).

\section{ENDNOTES}

${ }^{1}$ Baseline RSA did not predict orienting behavior, $R^{2}=.07, F(1,24)=1.7, p=.21$, nor self-regulatory abilities, $R^{2}=.05, F$ $(1,24)=1.32, p=.26$, in 1-month-old infants. In addition, we did not find statistical significant results when considering a two-way interaction low-intensity and high-intensity $R S A, R^{2}=.23, F(2,24)=3.19, p=.06$, or when considering a three-way interaction including baseline RSA, low-intensity and high-intensity $\operatorname{RSA}, R^{2}=.48, F(3,24)=2.2, p=.13$.

\section{ORCID}

Sara Cruz iD http://orcid.org/0000-0003-0011-7746

Fernando Ferreira-Santos (iD http://orcid.org/0000-0002-2656-5291

\section{REFERENCES}

Allen, J. J. B., Chambers, A. S., \& Towers, D. N. (2007). The many metrics of cardiac chronotropy: A pragmatic primer and a brief comparison of metrics. Biological Psychology, 74, 243-262.

Als, H. (1986). A synactive model of neonatal behavioral organization: Framework for the assessment of neurobehavioral development in the premature infant and for support of infants and parents in the neonatal intensive care environment. Physical \& Occupational Therapy in Pediatrics, 6, 3-53.

Als, H., Butler, S., Kosta, S., \& McAnulty, G. (2005). The Assessment of Preterm Infants' Behavior (APIB): Furthering the understanding and measurement of neurodevelopmental competence in preterm and full-term infants. Mental Retardation and Developmental Disabilities Research Reviews, 11, 94-102.

Als, H., Tronick, E., Lester, B., \& Brazelton, T. B. (1977). The Brazelton Neonatal Behavioral Assessment Scale (BNBAS). Journal of Abnormal Child Psychology, 5, 215-229.

Bazhenova, O. V., Plonskaia, O., \& Porges, S. W. (2001). Vagal reactivity and affective adjustment in infants during interaction challenges. Child Development, 72, 1314-1326.

Beauchaine, T. (2001). Vagal tone, development, and Gray's motivational theory: Toward an integrated model of autonomic nervous system functioning in psychopathology. Development and Psychopathology, 13, 183-214.

Berntson, G. G., Bigger, J. T. Jr., Eckberg, D. L., Grossman, P., Kaufmann, P. G., Malik, M., ... van der Molen, M. W. (1997). Heart rate variability: origins, methods, and interpretive caveats. Psychophysiology, 34, 623-648. 
Brazelton, T. B., \& Nugent, J. K. (1995). Neonatal behavioral assessment scale. London: Cambridge University Press.

Bryant, G. A., \& Barrett, H. C. (2007). Recognizing intentions in infant-directed speech evidence for universals. Psychological Science, 18, 746-751.

Calkins, S. D. (1997). Cardiac vagal tone indices of temperamental reactivity and behavioral regulation in young children. Developmental Psychobiology, 31, 125-135.

Calkins, S. D., Fox, N. A., \& Marshall, T. R. (1996). Behavioral and physiological antecedents of inhibited and uninhibited behavior. Child Development, 67, 523-540.

Calkins, S. D., Graziano, P. A., \& Keane, S. P. (2007). Cardiac vagal regulation differentiates among children at risk for behavior problems. Biological Psychology, 74, 144-153.

Clarkson, M. G., \& Berg, W. K. (1983). Cardiac orienting and vowel discrimination in newborns: Crucial stimulus parameters. Child Development, 54, 162-171.

Cruz, S., Crego, A., Ribeiro, E., Goncalves, O., \& Sampaio, A. (2015). A VEP study in sleeping and awake one-month-old infants and its relation with social behavior. International Journal of Developmental Neuroscience, 41, 37-43.

Davis, C. M., Crowell, D. H., \& Chun, B. J. (1965). Monophasic heart rate accelerations in human infants to peripheral stimulation. American Psychologist, 20, 478.

Fernald, A. (1993). Approval and disapproval: Infant responsiveness to vocal affect in familiar and unfamiliar languages. Child Development, 64, 657-674.

Field, T. M., Dempsey, J. R., Hatch, J., Ting, G., \& Clifton, R. K. (1979). Cardiac and behavioral responses to repeated tactile and auditory stimulation by preterm and term neonates. Developmental Psychology, 15, 406.

Figueras, F., Cruz-Martinez, R., Sanz-Cortes, M., Arranz, A., Illa, M., Botet, F., .. Gratacos, E. (2011). Neurobehavioral outcomes in preterm, growth-restricted infants with and without prenatal advanced signs of brain-sparing. Ultrasound in Obstetrics \& Gynecology, 38, 288-294.

Grossmann, T., Striano, T., \& Friederici, A. D. (2005). Infants' electric brain responses to emotional prosody. Neuroreport, 16, 1825-1828.

Hastings, P. D., Nuselovici, J. N., Utendale, W. T., Coutya, J., McShane, K. E., \& Sullivan, C. (2008). Applying the polyvagal theory to children's emotion regulation: Social context, socialization, and adjustment. Biological Psychology, 79, 299-306.

Hill, L. K., Hu, D. D., Koenig, J., Sollers, J. J. 3rd, Kapuku, G., ... Thayer, J. F. (2015). Ethnic differences in resting heart rate variability: A systematic review and meta-analysis. Psychosomatic Medicine, 77, 16-25.

Kushnerenko, E., Tomalski, P., Ballieux, H., Ribeiro, H., Potton, A., Axelsson, E. L., .. Moore, D. G. (2013). Brain responses to audiovisual speech mismatch in infants are associated with individual differences in looking behaviour. European Journal of Neuroscience, 38, 3363-3369.

Lester, B. M., Als, H., \& Brazelton, T. B. (1982). Regional obstetric anesthesia and newborn behavior: A reanalysis toward synergistic effects. Child Development, 53, 687-692.

Lewis, G. F., Stanfill, S., Zageris, D., Davila, M., \& Porges, S. W. (2011). Neural regulation of sensory gating in the auditory periphery: Relationship with listening and heart rate dynamics. Boston, MA: Society for Psychophysiological Research.

Lundqvist, C., \& Sabel, K. G. (2000). Brief report: The Brazelton Neonatal Behavioral Assessment Scale detects differences among newborn infants of optimal health. Journal of Pediatric Psychology, 25, 577-582.

Perez-Pereira, M., Fernandez, P., Gomez-Taibo, M., Gonzalez, L., Trisac, J. L., Casares, J., \& Dominguez, M. (2013). Neurobehavioral development of preterm and full term children: Biomedical and environmental influences. Early Human Development, 89, 401-409.

Porges, S. W. (1992). Autonomic regulation and attention. In B. A. Campbell, H. Hayne, \& R. Richardson (Eds.), Attention and information processing in infants and adults (pp. 201-223). Hillsdale, NJ: Erlbaum.

Porges, S. W. (1995). Orienting in a defensive world: Mammalian modifications of our evolutionary heritage. A polyvagal theory. Psychophysiology, 32, 301-318.

Porges, S. W. (2001). The polyvagal theory: Phylogenetic substrates of a social nervous system. International Journal of Psychophysiology, 42, 123-146.

Porges, S. W. (2003). The polyvagal theory: Phylogenetic contributions to social behavior. Physiology \& Behavior, 79 , 503-513.

Porges, S. W. (2011). The polyvagal theory: Neurophysiological foundations of emotions, attachment, communication, and selfregulation (Norton Series on Interpersonal Neurobiology). New York, NY: WW Norton \& Company.

Porges, S. W., Arnold, W. R., \& Forbes, E. J. (1973). Heart rate variability: An index of attentional responsivity in human newborns. Developmental Psychology, 8, 85.

Porges, S. W., \& Furman, S. A. (2011). The early development of the autonomic nervous system provides a neural platform for social behaviour: A polyvagal perspective. Infant and Child Development, 20, 106-118. 
Porges, S. W., \& Lewis, G. F. (2010). 2-The polyvagal hypothesis: Common mechanisms mediating autonomic regulation, vocalizations and listening. Handbook of Behavioral Neuroscience, 19, 255-264.

Porges, S. W., Macellaio, M., Stanfill, S. D., McCue, K., Lewis, G. F., Harden, E. R., ... Heilman, K. J. (2013). Respiratory sinus arrhythmia and auditory processing in autism: Modifiable deficits of an integrated social engagement system?. International Journal of Psychophysiology, 88, 261-270.

Posner, M. I. (1995). Attention in cognitive neuroscience: An overview. In M. S. Gazzaniga (Ed.), The cognitive neurosciences (pp. 615-624). Cambridge, MA: The MIT Press.

Propper, C., \& Moore, G. A. (2006). The influence of parenting on infant emotionality: A multi-level psychobiological perspective. Developmental Review, 26, 427-460.

Richards, J. E. (2001). Attention in young infants: A developmental psychophysiological perspective. In C. A. Nelson \& Luciana (Eds.), Handbook of developmental cognitive neuroscience (pp. 321-338). Cambridge, MA: MIT Press.

Rueda, M. R., Posner, M. I., \& Rothbart, M. K. (2004). Attentional control and self-regulation. Handbook of Self-Regulation: Research, Theory, and Applications, 2, 284-299.

Santesso, D. L., Schmidt, L. A., \& Trainor, L. J. (2007). Frontal brain electrical activity (EEG) and heart rate in response to affective infant-directed (ID) speech in 9-month-old infants. Brain and Cognition, 65, 14-21.

Schmidt, L. A., \& Segalowitz, S. J. (2008). Developmental psychophysiology: Theory, systems, and methods. New York: Cambridge University Press.

Sprangler, G., Fremmer-Bombik, E., \& Grossmann, K. (1996). Social and individual determinants of infant attachment security and disorganization. Infant Mental Health Journal, 17, 127-139.

Vranekovic, G., Hock, E., Isaac, P., \& Cordero, L. (1974). Heart rate variability and cardiac response to an auditory stimulus. Biology of the Neonate, 24, 66-73.

How to cite this article: Cruz S, Ferreira-Santos F, Oliveira-Silva P, Ribeiro E, Gonçalves Ó, Sampaio A. Vagal modulation of 1-month-old infants to auditory stimuli is associated with self-regulatory behavior. Social Development. 2018;27:322-334. https://doi.org/10.1111/sode.12270 